\title{
Canadian Thoracic Society reborn
}

\section{Renaissance de la Société canadienne de thoracologie}

$\mathrm{T}$ he past weekend (April 27 to 29) in Ottawa, Ontario, I attended a stand-alone meeting of the Canadian Thoracic Society (CTS) for the first time in many years. The other recent meetings of the CTS that I have attended were all held in conjunction with the annual meeting of the American College of Chest Physicians, usually in the United States. The Ottawa meeting was a success so far as I am concerned, but I think the big news is that this will be the start of a new tradition.

For several years the CTS leadership has been pointing toward an annual meeting of the CTS in

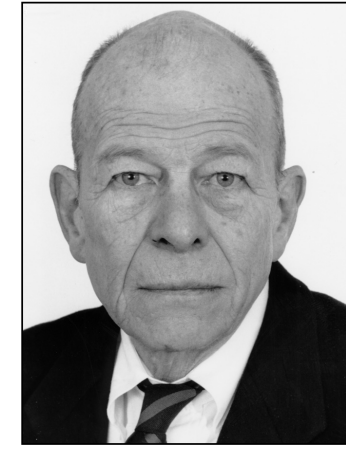

Nick R Anthonisen Canada. A series of events has made this feasible at present. First, the Canadian Lung Association has acquired visionary new leadership and is actively developing a 'framework' for planning its activities in the support of lung health. The Canadian Lung Association and the CTS have worked harmoniously to develop the framework and it is on track. Each organization is represented on the other's board, and each believes the other to be an asset. Second, the CTS is being reorganized under the current leadership of Darcy Marciniuk with rules of governance and a comprehensive committee structure. Third, the CTS has acquired new resources so that it can pay its own way. Plans are under way to substantially bolster its administrative support, a vital requirement. Fourth, a recent CTS President, Gerry Cox, realized that various CTS members met through the year to conduct their activities, including housekeeping, and that if these were gathered into the same place at the same time, the nucleus of a meeting would be present. This vision was illustrated at the recent meeting. Immediately before the CTS meeting, the Canadian Lung Association met to further develop its framework and was graced by the attendance of two federal cabinet ministers. The CTS Board and its subcommittees (Long Range Planning, Membership and Industry Advisory) all met. Directors of the specialty training programs met. In addition, the annual Residents-Fellows research paper competition was held. Finally, there was a well-attended Sleep Apnea Summit, which will likely produce a summary for publication in the Canadian Respiratory Journal. I attended a session of the research paper competition and enjoyed it thoroughly, being impressed with both the work presented and the skill of the judges in producing lively discussions.

Bigger things are to come next year, with a meeting in Montreal, Quebec, in late June (tentatively June 18 to 22). In addition to the activities noted above, the Canadian COPD Alliance will attend and hold its meeting, as will the Canadian Respiratory Health Professionals Society. The annual refresher course for residents about to take the Royal College examinations will also be part of the meeting. The strategy of consolidating established annual meetings of the respiratory
T a fin de semaine dernière (du 27 au 29 avril), à LOttawa, j'ai assisté pour la première fois depuis très longtemps à une assemblée indépendante de la Société canadienne de thoracologie (SCT). Les autres réunions de la SCT auxquelles j'avais assisté ces dernières années avaient toutes eu lieu en concomitance avec l'assemblée annuelle de l'American College of Chest Physicians, le plus souvent aux États-Unis. L'assemblée qui s'est tenue à Ottawa a été, quant à moi, une réussite, mais je crois que la grande nouvelle, c'est qu'elle marquera le début d'une nouvelle tradition.

Depuis plusieurs années, la direction de la SCT a comme objectif de tenir une assemblée annuelle de la SCT au Canada. Une suite d'événements a, cette fois-ci, rendu la chose possible. Premièrement, l'Association pulmonaire du Canada (APC) a à sa tête de nouveaux dirigeants visionnaires et elle poursuit activement l'élaboration d'un cadre pour la planification de ses activités visant l'amélioration de la santé pulmonaire. Fruit d'une collaboration harmonieuse entre l'APC et la SCT, l'élaboration de ce cadre est dans la bonne voie. Chacune des organisations est représentée au conseil d'administration de l'autre et considère l'autre comme un atout. Deuxièmement, une réorganisation de la SCT est présentement en cours sous la direction de Darcy Marciniuk et s'accompagne de règles de saine gestion et d'une structure détaillée de comités. Troisièmement, la SCT a obtenu de nouvelles ressources de sorte qu'elle peut maintenant couvrir ses frais. On prévoit également prendre des mesures pour améliorer considérablement le soutien administratif de la SCT, un besoin vital. Quatrièmement, Gerry Cox, l'un des derniers présidents de la SCT, s'était rendu compte que des membres de la SCT se réunissaient durant l'année pour mener leurs activités, y compris pour les affaires courantes, et que, si l'on rassemblait toutes ces personnes en même temps, au même endroit, on obtiendrait un embryon d'assemblée. La vision s'est concrétisée à la dernière assemblée. Juste avant l'assemblée de la SCT, l'APC s'est réunie pour poursuivre l'élaboration de son cadre et deux ministres du Cabinet fédéral lui ont fait l'honneur de leur présence. Le conseil d'administration de la SCT et ses souscomités (planification à long terme, adhésion, sous-comité consultatif de l'industrie) se sont tous réunis. Les directeurs des programmes de spécialisation ont aussi tenu une réunion. À cela s'ajoute le concours annuel de présentation de rapports de recherche des résidents et des associés. Enfin, il y a eu un sommet fort couru, celui sur les apnées du sommeil, qui débouchera probablement sur un résumé à publier dans la Revue canadienne de pneumologie. J'ai assisté à une séance du concours de présentation de rapports de recherche, et cela m'a vraiment plu; j'ai été impressionné tant par les travaux présentés que par l'habileté des juges à susciter des discussions animées.

De grandes choses s'en viennent l'an prochain, à l'occasion de l'assemblée prévue à Montréal, à la fin de juin (provisoirement du 18 au 22 juin). En plus des activités déjà mentionnées, l'Alliance canadienne sur la MPOC sera présente et tiendra sa réunion, ce 
community into one time and place will produce a whole that is greater than the sum of its parts. It seems to me that the Montreal meeting will be a success based only on commitments already in hand. Obviously, the long-term idea is to have an annual recurring Canadian meeting of the CTS and allied groups. This is a noble objective, and I was delighted to have been able to observe its early development.

It must be noted that there is much more to the CTS than a new meeting. It has been a leader in developing therapeutic guidelines, including those for asthma, COPD and sleep-related disorders. It is also a leader in developing methods for guideline implementation, and boasts an important and dedicated figure in this area in Louis-Philippe Boulet. Further, over the years, it has developed a fruitful association with the American College of Chest Physicians that both organizations are anxious to continue. The future looks rosy, and I am glad.

Nick R Anthonisen MD Editor-in-Chief, Canadian Respiratory Journal que feront également les Professionnels canadiens en santé respiratoire. Le cours annuel de mise à jour destiné aux résidents qui s'apprêtent à passer l'examen du Collège royal sera aussi à l'ordre du jour de la réunion. La stratégie qui consiste à tenir en concomitance et au même endroit les assemblées annuelles bien établies du milieu de la santé pulmonaire produira un tout plus grand que la somme de ses parties. À en juger simplement par les engagements déjà confirmés, j'ai l'impression que l'assemblée de Montréal sera un succès. Évidemment, l'objectif à long terme est de tenir systématiquement tous les ans, au Canada, une assemblée de la SCT et de ses groupes affiliés. C'est là un objectif noble, et j'ai été ravi d'avoir été témoin des premiers pas en ce sens.

Il convient de souligner que la SCT est loin de se résumer à la tenue d'une nouvelle assemblée. La SCT est un chef de file dans l'élaboration de lignes directrices sur le traitement de diverses affections, dont l'asthme, la MPOC et les troubles du sommeil. Elle joue aussi un rôle prépondérant dans l'élaboration de méthodes de mise en œuvre des lignes directrices et compte sur une personne importante et dévouée dans ce domaine, M. Louis-Philippe Boulet. En outre, la SCT a su former au fil des ans, avec l'American College of Chest Physicians, une association fructueuse que les deux organisations tiennent beaucoup à poursuivre. L'avenir se présente bien, et j'en suis très heureux.

Nick R. Anthonisen, M.D. Rédacteur en chef, Revue canadienne de pneumologie 


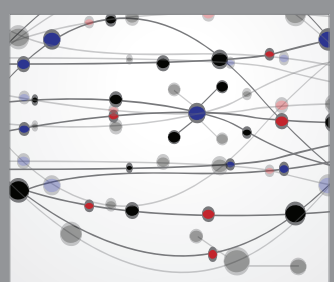

The Scientific World Journal
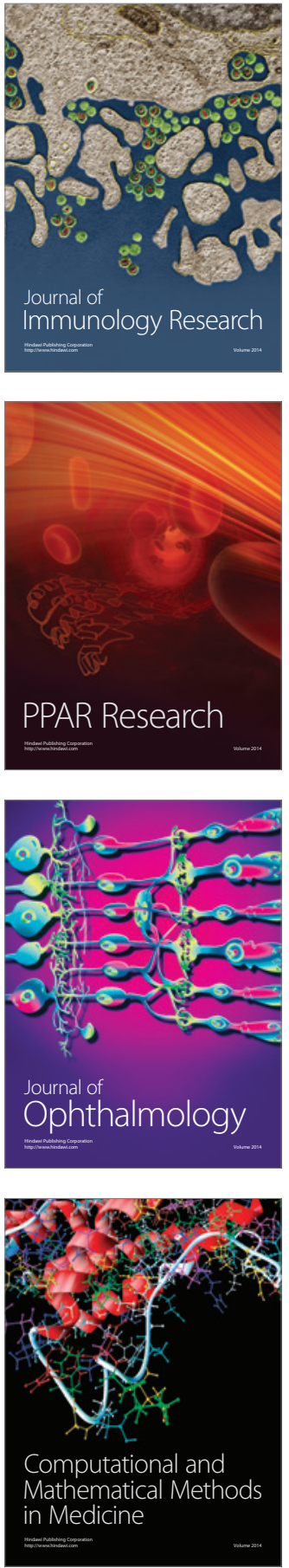

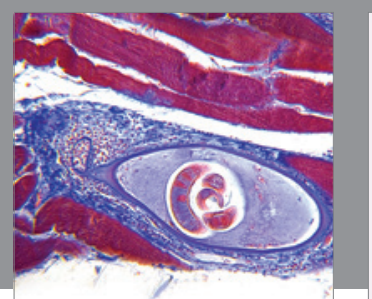

Gastroenterology Research and Practice

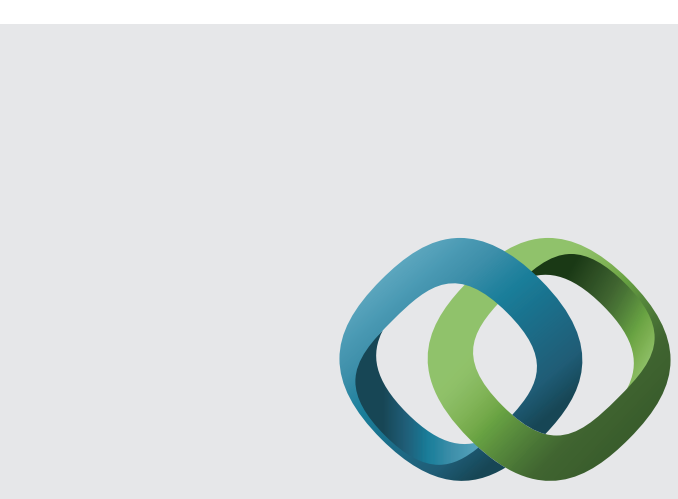

\section{Hindawi}

Submit your manuscripts at

http://www.hindawi.com
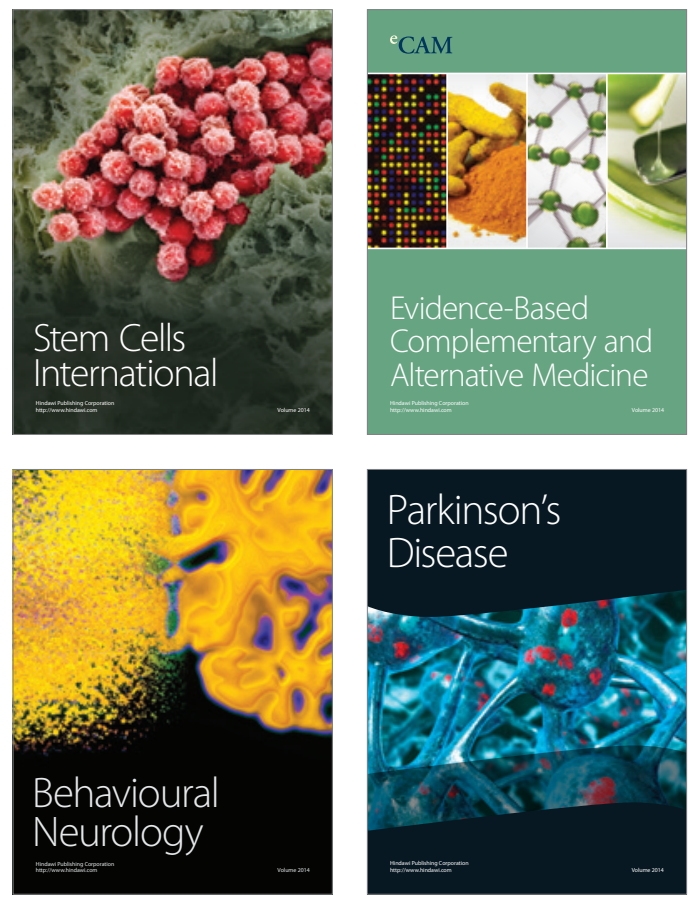
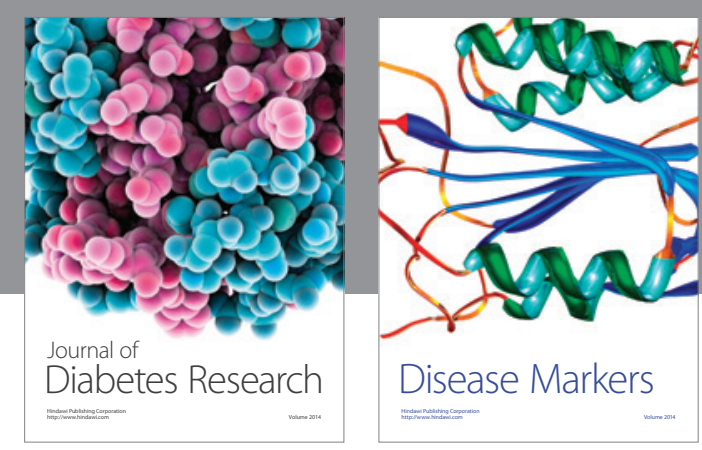

Disease Markers
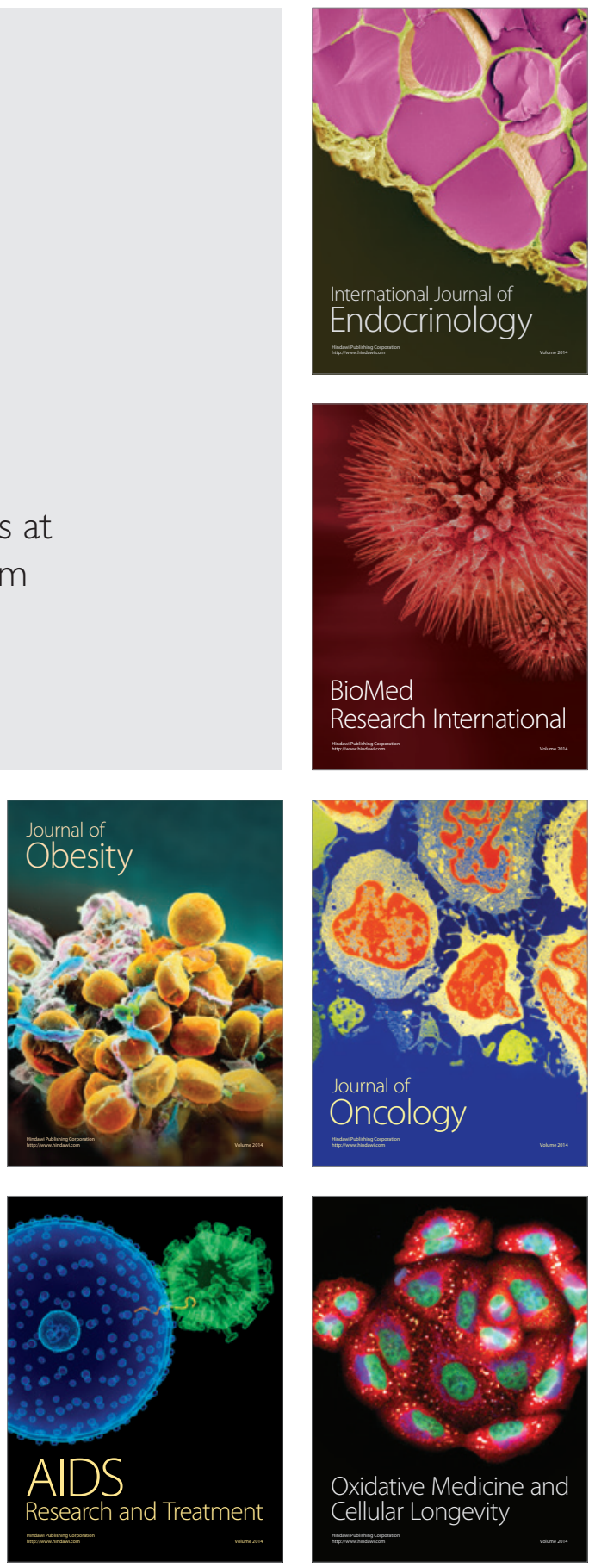